\title{
DIABETES \\ Extended genetic testing improves MODY diagnosis
}

Twice as many patients with maturityonset diabetes of the young (MODY) would receive a correct diagnosis if the criteria for genetic screening were expanded, suggest the results of a new study published in Diabetes Care.

An estimated $80 \%$ of patients with MODY are incorrectly diagnosed as having type 1 or type 2 diabetes mellitus (T1DM or T2DM, respectively). Typically, patients are only referred for screening if they are aged $<25$ years at diagnosis, have a parental history of diabetes mellitus and are noninsulin dependent.

In a group of 247 patients diagnosed as having T1DM, residual $\beta$-cell function was found in 20 patients. Among 322 patients diagnosed as having T2DM, 80 were not insulin resistant or had an age of disease onset $<30$ years. Patients with these features atypical of T1DM and T2DM were tested for mutations in the HNF1 homeobox A and hepatocyte nuclear factor $4 a$ genes. Mutations confirming MODY were found in 14 patients. The glucokinase gene was sequenced in 40 patients who had mild fasting hyperglycaemia and one person, initially diagnosed as having T2DM, was found to have MODY caused by a mutation in this gene.

"We tried to choose features that would be generally available in the clinic, so we used metabolic syndrome as a marker for insulin resistance and a random C-peptide measurement taken at the clinic to assess $\beta$-cell function," says senior researcher Katharine Owen. "We suggest that those patients diagnosed under age 30 who are C-peptide positive should be considered for MODY testing."

\section{Fiona Mitchell}

Original article Thanabalasingham, G. et al. Systematic assessment of etiology in adults with a clinical diagnosis of young-onset type 2 diabetes is a successful strategy for identifying maturity-onset diabetes of the young. Diabetes Care doi:10.2337/dc11-1243 